\title{
A High-Impedance Ground Plane Applied to a Cellphone Handset Geometry
}

\author{
Romulo F. Jimenez Broas, Daniel F. Sievenpiper, Member, IEEE, and Eli Yablonovitch, Fellow, IEEE
}

\begin{abstract}
A high-impedance electromagnetic surface is a new type of metallic structure exhibiting high surface impedance and the suppression of propagating surface currents at a particular frequency band. We experimentally characterize such a high-impedance surface designed near $2.4 \mathrm{GHz}$. We describe an antenna built on such a surface, integrated into a printed circuit board that was designed for the form factor of a portable handset. Measurement shows high radiation efficiency near $2.4 \mathrm{GHz}$.
\end{abstract}

Index Terms-Antennas, bandgap, coupling, handset communications, surface impedance, surface waves.

\section{INTRODUCTION}

$\mathbf{R}$ ECENTLY, a new kind of ground plane has been developed that is capable of suppressing the propagation of radio-frequency surface currents [1]. This new surface is important for antenna applications because it is has the property of partially isolating the radiating elements from the nearby electromagnetic surroundings. For example, the property of surface current suppression can be very useful to portable telephony, where current handset telephone designs radiate nearly one-half of the emitted power into the user's head [2]. While the biological implications of this are yet to be determined, the absorption by the user's head decreases the radiation efficiency by roughly $3 \mathrm{~dB}$. By shielding the antenna from the user, this radiation efficiency can be greatly increased, resulting in greater battery life, which, in turn, affects the weight of the phone.

The new surface also provides a high electromagnetic surface impedance, which allows the antenna to lie directly adjacent to the ground plane without being shorted out. This allows compact antenna designs where the radiating elements are confined to limited spaces. Hence, the two properties described above result in a low-profile antenna that can fit into the small form factor of a cordless handset, while also partially shielding the antenna from the effects of the user. As an example, we have built an antenna using a high-impedance electromagnetic surface as a ground plane, and integrated it into the circuit board of a portable handset. Measurement shows the antenna achieves high radiation efficiency, even in the presence of a lossy dielectric body.

Manuscript received June 12, 2000.

R. F. Jimenez Broas is with the Electromagnetic Systems Laboratory, Raytheon Systems Company, El Segundo, CA 90245 USA.

D. F. Sievenpiper is with HRL Laboratories LLC, Malibu, CA 90265 USA.

E. Yablonovitch is with the Department of Electrical Engineering, University

of California at Los Angeles, Los Angeles, CA 90032 USA.

Publisher Item Identifier S 0018-9480(01)05051-7.

\section{High-IMPEDANCE ELECTROMAGNETIC SURFACE}

The high-impedance electromagnetic surface is made of continuous metal, and it conducts dc currents, but it does not conduct ac currents within a forbidden frequency band. The physical geometry consists of a metal sheet, textured with a two-dimensional lattice of resonant elements, which act as a two-dimensional filter to prevent the propagation of electric currents [3].

The high-impedance properties occur near the resonance frequency of the surface $\omega=1 / \sqrt{L C}$, where $L$ is the effective sheet inductance and $C$ is the effective sheet capacitance. The high-impedance electromagnetic surface studied is shown in Fig. 1. It consists of a three-layer printed circuit board, in which the lowest layer is solid metal and the top two layers contain nearly hexagonal metal patches. The inductance of the surface is related to the thickness of the circuit board by $L=\mu t$, where $\mu$ is the magnetic permeability and $t$ is the thickness of the board. For a standard 1.6-mm circuit board, the sheet inductance is $2.01 \mathrm{nH} / \mathrm{square}$. To produce a bandgap in the $2.4-\mathrm{GHz}$ frequency range, a capacitance of $2.19 \mathrm{pF}$. square is needed. This high capacitance is obtained by using two parallel metal plates with a thin insulating layer between them. The patches are roughly $5-\mathrm{mm}$ wide, and are connected to the ground plane by plated metal vias. The two layers of patches are separated by $0.05 \mathrm{~mm}$ of polyimide insulator $(\varepsilon \approx 3.25)$. The patches in the lower layer are separated from the solid metal layer by a 1.6-mm substrate made of standard fiberglass printed-circuitboard material, commonly known as FR4 $(\varepsilon \approx 4.5)$. The overlap between neighboring patches provides a sheet capacitance of about $2.13 \mathrm{pF}$ - square. The structure shown in Fig. 1 has a resonance frequency of $f=1 / 2 \pi \sqrt{L C}=2.43 \mathrm{GHz}$.

The width of the bandgap can be shown to be $\Delta f / f=$ $\sqrt{L C} / \sqrt{\mu_{0} / \varepsilon_{0}}=8.14 \%$, which translates to a bandgap frequency range of $2.33-2.53 \mathrm{GHz}$. In this frequency range, the electromagnetic surface exhibits high surface impedance, does not conduct ac currents, and suppresses the propagation of radio-frequency surface waves. For portable communications, the suppression of surface waves can improve performance by effectively confining antenna radiation to only one hemisphere, acting as a shield between the antenna and the user.

A TE surface-wave measurement using current loop probe antennas was performed [3]. In TE surface waves, the electric field is parallel to the surface, and the magnetic field forms vertical loops that arc out of the surface. They can be measured using small loop antennas, as shown in Fig. 2. This type of probe 




(a)

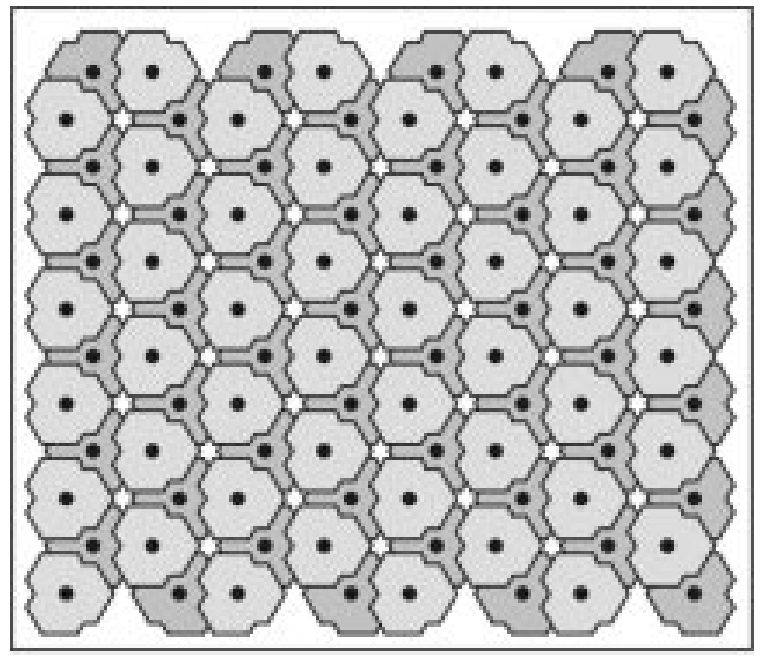

(b)

Fig. 1. (a) Side view of the high-impedance electromagnetic surface. (b) Top view of the high-impedance electromagnetic surface.



Fig. 2. Experimental setup to measure the transmission of TE surface waves.

can be constructed as a small wire loop connecting the two conductors of a coaxial cable. The plane of the loop is parallel to the surface, creating a vertical magnetic field that couples to the magnetic field of the TE surface waves.

The result of the measurement is shown in Fig. 3, confirming the bandgap. A sharp jump in transmission of about $20 \mathrm{~dB}$ occurs at $2.53 \mathrm{GHz}$, indicating the TE band edge. Beyond this frequency, transmission is much higher compared to the bandgap frequency band, with only small fluctuations due to speckle. Since the probe used in this experiment can also couple to TM waves, the TM band edge is also visible in this measurement at about $2.3 \mathrm{GHz}$.

\section{Cordless Handset PRototyPe-Measurement AND ANALYSIS}

The antenna geometry that we studied consisted of a horizontal copper wire etched on a piece of FR4 board. This antenna geometry was a bent-wire horizontal antenna fabricated as an additional printed circuit layer. An impedance-matching



Fig. 3. Measurement of the transmission of TE surface waves.

pattern was employed on the end near the feed point to ensure low reflection $\left(S_{11}\right)$ over a wide band.

The high-impedance electromagnetic surface with the attached antenna was soldered to a $5 \mathrm{~cm} \times 15 \mathrm{~cm}$ FR4 circuit board to simulate the presence of the phone circuitry. This prototype simulates a design in which the antenna is integrated into the circuit board of the phone to minimize cost. One side of the circuit board was solid copper, and the other side etched clean to reveal bare FR4. The solid metal back side of the antenna ground plane was soldered to the copper side of the blank circuit board. The addition of real circuit traces and components may tend to increase absorption into the board, thus, this may be considered a best-case situation. The antenna 




Fig. 4. Portable handset prototype with an antenna using a high-impedance electromagnetic surface as a ground plane.



Fig. 5. Measured $S 11$ plot of the antenna using a high-impedance electromagnetic surface as a ground plane.

geometry along with the prototype is shown in Fig. 4. The $S 11$ of the antenna and prototype is shown in Fig. 5.

The radiation pattern of the antenna on a high-impedance electromagnetic surface is shown in Fig. 6. The frequency shown is $2.44 \mathrm{GHz}$, which is inside the bandgap. At the $0^{\circ}$ polar angle, the cordless telephone prototype has its antenna facing the horn and its attached FR4 Circuit Board pointing toward the $270^{\circ}$ polar angle. Note that the front-to-back ratio is approximately $10 \mathrm{~dB}$. Antenna measurements were performed from 2.2 to $2.8 \mathrm{GHz}$. Since the designed bandgap is from 2.33 to $2.53 \mathrm{GHz}$, frequencies both inside and outside the bandgap were analyzed.

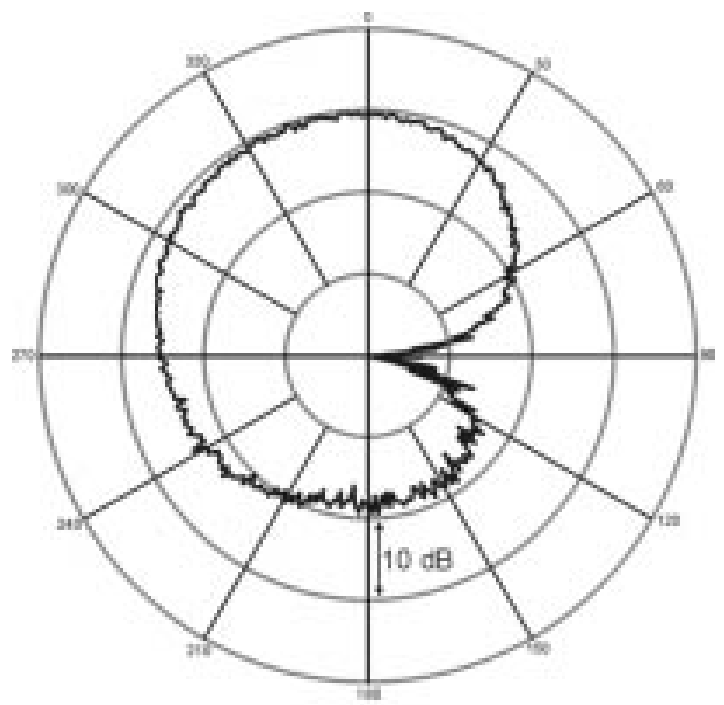

Fig. 6. Radiation pattern of an antenna on a high-impedance electromagnetic surface. The frequency is $2.44 \mathrm{GHz}$.



Fig. 7. Radiation efficiency versus frequency.

The radiation efficiency as a function of frequency shown in Fig. 7 was obtained by comparing the radiation pattern of the antenna on a high-impedance electromagnetic surface to a standard gain horn whose radiation properties were well documented [4]. The radiation transmissions of both the antenna on a high-impedance electromagnetic surface and the standard gain horn were measured. The first steps in the calculation are obtaining the cross sections associated with the full-width at halfmaximum (FWHM) values for both radiation transmissions. If $\Omega_{H}$ is the standard gain horn's cross section and $\Omega_{A}$ is the cross section of the antenna on a high-impedance electromagnetic surface, then antenna efficiency $\eta$ is defined as follows:

$$
\eta=\frac{\Omega_{A}}{\Omega_{H}}\left(10^{-\Delta / 10}\right)
$$

where $\Delta$ is the absolute value difference between the peak transmission values of the horn and antenna. The antenna efficiency definition above compares the efficiency of the antenna on a high-impedance electromagnetic surface to the efficiency of the horn. 
The peak antenna efficiency occurs at $2.44 \mathrm{GHz}$, which is also the near the center of the designed bandgap of the highimpedance ground plane. For frequencies near the center of the bandgap, the antenna efficiencies are quite high (above $80 \%$ ). For frequencies outside the bandgap, however, the antenna efficiencies decrease below $50 \%$.

This result can be explained as follows. Within the bandgap, the suppression of surface currents tends to confine the radiation to the forward hemisphere and limit the interaction of the antenna with its electromagnetic surroundings. For frequencies outside the bandgap, the antenna couples strongly to the nearby electromagnetic surroundings, including the attached circuit board. Significant energy leaks away as induced currents in the circuit board, and is then absorbed. Hence, it eventually becomes lost radiation and decreases the antenna efficiency.

\section{CONCLUSION}

High-impedance electromagnetic surfaces have two noteworthy characteristics over a designed frequency band that makes it an ideal choice for antenna ground planes. Its ability to suppress the propagation of surface currents allows it to partially isolate the radiating elements from the nearby electromagnetic surroundings. The surface also has a high electromagnetic surface impedance that allows the radiating elements to be placed very close to the ground plane. These two properties were utilized when we built a compact low-profile antenna integrated to a small cordless handset while the antenna itself was shielded from the user. The peak antenna efficiencies were inside the bandgap of the high-impedance electromagnetic surface.

\section{ACKNOWLEDGMENT}

The work described in this paper was performed when all of the authors were with the Department of Electrical Engineering, University of California at Los Angeles (UCLA).

\section{REFERENCES}

[1] D. Sievenpiper, L. Zhang, R. F. Jimenez Broas, N. Alexopolous, and E. Yablonovitch, "High-impedance electromagnetic surfaces with a forbidden frequency band," IEEE Trans. Microwave Theory Tech., vol. 47, pp. 2059-2074, Nov. 1999.

[2] M. Jensen and Y. Rahmat-Samii, "EM interaction of handset antennas and a human in personal communications," Proc. IEEE, vol. 83, pp. 7-17, Jan. 1995

[3] D. Sievenpiper, "High-impedance electromagnetic surfaces," Ph.D. dissertation, Dept. Elect. Eng., Univ. California at Los Angeles, Los Angeles, CA, 1999.
[4] R. F. Jimenez Broas, "Experimental characterization of high impedance electromagnetic surfaces in the microwave frequency regime," M.S. thesis, Dept. Elect. Eng., Univ. California at Los Angeles, Los Angeles, CA, 1999.

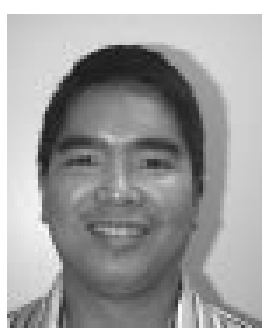

Romulo F. Jimenez Broas received the A.B. degree in physics from the University of California at Berkeley, in 1996 and the M.S. degree in electrical engineering from the University of California at Los Angeles, in 1999.

$\mathrm{He}$ is currently an Electrical Engineer with the Electromagnetic Systems Laboratory, Raytheon Systems Company, El Segundo, CA. His current interests are in semiconductor devices, antennas, guided waves, and scanning arrays.



Daniel F. Sievenpiper (S'95-M'98) received the B.S. and Ph.D. degrees in electrical engineering from the University of California at Los Angeles, in 1994 and 1999, respectively.

$\mathrm{He}$ is currently a Research Staff Member at HRL Laboratories LLC, Malibu, CA. He has authored eight journal publications. He has ten pending U.S. patents. His research included photonic crystals, high-impedance electromagnetic surfaces, and novel antennas.

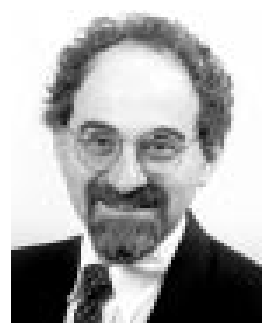

Eli Yablonovitch (M'75-SM'90-F'92) received the Ph.D. degree in applied physics from Harvard University, Cambridge, MA, in 1972.

He was with Bell Telephone Laboratories for two years. He then became a Professor of applied physics at Harvard University. In 1979, he joined the Exxon Corporation to perform research on photovoltaic solar energy. In 1984, he joined Bell Communications Research, where he was a Distinguished Member of Staff and Director of Solid-State Physics Research. In 1992, he joined the University of California, Los Angeles, where he is currently a Professor of electrical engineering. His research has covered a broad range of topics including nonlinear optics, laser-plasma interaction, infrared laser chemistry, photovoltaic energy conversion, strained-quantum-well lasers, and chemical modification of semiconductor surfaces. His current main interests are in optoelectronics, high-speed optical communications, high-efficiency light-emitting diodes and nanocavity lasers, photonic crystals at optical and microwave frequencies, quantum computing, and quantum communication.

Dr. Yablonovitch is a member of Eta Kappa Nu and a Fellow of the American Physical Society and the Optical Society of America. He chaired the 1979 Gordon Conference on Nonlinear Optics and Lasers. He was the recipient of the Alfred P. Sloan Fellowship (1978-1979), the Adolf Lomb Medal of the Optical Society of America (1978), an Research and Development 100 Award (1990), the W. Streifer Scientific Achievement Award of the IEEE/Lasers and Electro-Optics Society (LEOS) (1993), and the R. W. Wood Prize of the Optical Society of America (1996). 